\title{
GENERATING AND COGENERATING STRUCTURES
}

\author{
BY \\ JOHN A. BEACHY
}

\begin{abstract}
A functor $T: \mathscr{A} \rightarrow \mathscr{B}$ acts faithfully on the right of a class of objects $\mathscr{A}^{\prime}$ of $\mathscr{A}$ if it distinguishes morphisms out of objects of $\mathscr{A}^{\prime}$ (that is, $A^{\prime} \in \mathscr{A}^{\prime}, X \in \mathscr{A}$, $f, g \in \mathscr{A}\left(A^{\prime}, X\right)$ and $f \neq g$ implies $\left.T(f) \neq T(g)\right)$. We define a full subcategory $\mathscr{R} \mathscr{F}(T)$ such that $T$ acts faithfully on the right of the objects of $\mathscr{R} \mathscr{F}(T)$. An object $U \in \mathscr{A}$ is a generator if $H^{U}: \mathscr{A} \rightarrow \mathscr{E} n s$ is faithful, and if $H^{U}$ is not faithful, we may still consider $\mathscr{R} \mathscr{F}\left(H^{U}\right)$. This gives rise to the notion of a generating structure. Cogenerating structures are defined dually, and various canonical generating and cogenerating structures are defined for the category of $R$-modules. Relationships between these can be used in the homological classification of rings.
\end{abstract}

0. Introduction. If $\mathscr{A}$ and $\mathscr{B}$ are categories and $T: \mathscr{A} \rightarrow \mathscr{B}$ is a functor, either covariant or contravariant, we will say that $T$ acts faithfully on the right of an object $A \in \mathscr{A}$ if for all $X \in \mathscr{A}$ and $f, g \in \mathscr{A}(A, X), f \neq g$ implies $T(f) \neq T(g)$. Let $\mathscr{R} \mathscr{F}(T)$ denote the full subcategory of $\mathscr{A}$ determined by all objects $A \in \mathscr{A}$ on which $T$ acts faithfully on the right, if such objects exist. (The full subcategory $\mathscr{L} \mathscr{F}(T)$ is defined dually.) If $\mathscr{A}$ is sup-complete, then so is $\mathscr{R} \mathscr{F}(T)$ for any $T$ such that $\mathscr{R} \mathscr{F}(T)$ is nonempty. Conversely, if $\mathscr{A}^{\prime}$ is a full sup-complete subcategory of a locally small abelian category, then $\mathscr{A}^{\prime}=\mathscr{R} \mathscr{F}(T)$ for an idempotent preradical $T: \mathscr{A} \rightarrow \mathscr{A}$.

If $T: \mathscr{A} \rightarrow \mathscr{B}$ is covariant and has a left adjoint $S: \mathscr{B} \rightarrow \mathscr{A}$, then in the standard way the adjoint situation defines for each $A \in \mathscr{A}$ a morphism $\psi_{A}: S T(A) \rightarrow A$. We show that $A \in \mathscr{R} \mathscr{F}(T) \Leftrightarrow \psi_{A}$ is an epimorphism. Dual results are given for $\mathscr{L} \mathscr{F}(T)$.

In $\S 3$ we investigate $\mathscr{R} \mathscr{F}(T)$ for functors of the form $T=H^{U}$, for $U \in \mathscr{A}$. A generating structure is a class of objects $\mathscr{C}$ and a class of objects $\mathscr{D}$ such that $C \in \mathscr{C} \Leftrightarrow D \in \mathscr{R} \mathscr{F}\left(H^{C}\right)$, for all $D \in \mathscr{D}$, and $D \in \mathscr{D} \Leftrightarrow D \in \mathscr{R} \mathscr{F}\left(H^{C}\right)$ for all $C \in \mathscr{C}$. It is supported by $U \in \mathscr{A}$ if the full subcategory determined by $\mathscr{D}$ is $\mathscr{R} \mathscr{F}\left(H^{U}\right)$. Cogenerating structures are defined dually.

In $\S 4$ we specialize to the category ${ }_{R} \mathscr{M}$ of unital left $R$-modules over an associative ring $R$ with identity. We investigate the generating and cogenerating structures supported by ${ }_{R} R$, the injective envelope ${ }_{R} E(R)$ of ${ }_{R} R$, the direct sum ${ }_{R} K$ of

Received by the editors. May 5, 1970.

AMS 1970 subject classifications. Primary 18A05, 16A62; Secondary 18A35, 16A52.

Key words and phrases. $T$-faithful subcategory, idempotent preradical, radical, generating structure, cogenerating structure, fully divisible $R$-module, cofaithful $R$-module, torsionless $R$-module, faithful $R$-module, cogenerator ring, $S$-ring. 
representatives of each isomorphism class of simple modules, and the injective envelope ${ }_{R} E(K)$ of ${ }_{R} K$. These give rise to known classes of modules as well as to some new classes. Relations between these generating and cogenerating structures can be used to characterize self-injective rings, semisimple rings with minimum condition, semisimple rings, cogenerator rings, and several new classes of rings. These results follow from a single theorem (and its dual) describing relations between generating structures (cogenerating structures), so this theory allows a unified approach to a variety of questions. It is hoped that the notions of generating structure and cogenerating structure will prove to be useful in the homological classification of rings.

1. $T$-faithful subcategories. We will assume that the reader is familiar with the basic notions of category theory as developed in Mitchell [6]. For a category $\mathscr{A}$ we will usually write $A \in \mathscr{A}$ when in fact we mean that $A$ is an object of $\mathscr{A}$, and when the morphisms are obvious a category may be specified by giving only the objects. We will let $\mathscr{E} n s$ and $\mathscr{A} b$ denote the categories of sets and abelian groups, respectively. The definitions and results involving functors will include both the covariant and contravariant cases unless specifically restricted to one or the other.

Recall that a functor $T: \mathscr{A} \rightarrow \mathscr{B}$ is called faithful if for any $A, A^{\prime} \in \mathscr{A}$ and any pair of morphisms $f, g \in \mathscr{A}\left(A, A^{\prime}\right), f \neq g$ implies $T(f) \neq T(g)$. To generalize this, we make the following definition.

1.1. Definition. For a functor $T: \mathscr{A} \rightarrow \mathscr{B}$ and a class $\mathscr{A}^{\prime}$ of objects of $\mathscr{A}$, we say that $T$ acts faithfully on the right of $\mathscr{A}^{\prime}$ if for each $A^{\prime} \in \mathscr{A}^{\prime}$ and each $X \in \mathscr{A}$, $f, g \in \mathscr{A}\left(A^{\prime}, X\right)$ and $f \neq g$ implies $T(f) \neq T(g)$. Equivalently, we say that $\mathscr{A}^{\prime}$ is right $T$-faithful.

Dually, $\mathscr{A}^{\prime}$ is left $T$-faithful if for each $A^{\prime} \in \mathscr{A}^{\prime}$ and each $X \in \mathscr{A}, f, g \in \mathscr{A}\left(X, A^{\prime}\right)$ and $f \neq g$ implies $T(f) \neq T(g)$.

1.2. Definition. Let $T: \mathscr{A} \rightarrow \mathscr{B}$ be a functor. Let $\mathscr{R} \mathscr{F}(T)$ denote the full subcategory of $\mathscr{A}$ determined by all objects of $\mathscr{A}$ which are right $T$-faithful (if such objects exist). Let $\mathscr{L} \mathscr{F}(T)$ denote the full subcategory of $\mathscr{A}$ determined by all objects of $\mathscr{A}$ which are left $T$-faithful.

The functor $T$ is faithful if and only if $\mathscr{R} \mathscr{F}(T)=\mathscr{A}$ or $\mathscr{L} \mathscr{F}(T)=\mathscr{A}$, in which case $\mathscr{R} \mathscr{F}(T)=\mathscr{L} \mathscr{F}(T)$. In general, $\mathscr{R} \mathscr{F}(T)$ may be quite different from $\mathscr{L} \mathscr{F}(T)$, as the following example shows. Let $T: \mathscr{A} b \rightarrow \mathscr{A} b$ be the functor $T(A)=\operatorname{Hom}_{z}\left(Z_{2}, A\right)$. It can be seen that $\mathscr{L} \mathscr{F}(T)$ includes only the trivial abelian group, and it follows from later results that $A \in \mathscr{R} \mathscr{F}(T) \Leftrightarrow$ each element of $A$ has order two.

The following propositions investigate the properties of the subcategory $\mathscr{R} \mathscr{F}(T)$. Unless the dual statements concerning $\mathscr{L} \mathscr{F}(T)$ are of particular interest later, they will be omitted.

1.3. Proposition. Let $T: \mathscr{A} \rightarrow \mathscr{B}$ be a covariant functor.

(i) If $f: A \rightarrow A^{\prime}$ in $\mathscr{A}$ and $A^{\prime} \in \mathscr{R} \mathscr{F}(T)$, then if $T(f)$ is an epimorphism, so is $f$. 
(ii) If $D$ is the diagram

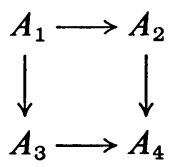

in $\mathscr{A}$, and $A_{1} \in \mathscr{R} \mathscr{F}(T)$, then if $T(D)$ is commutative, so is $D$.

Proof. (i) Let $g, h \in \mathscr{A}\left(A^{\prime}, X\right)$. If $g f=h f$, then $T(g) T(f)=T(h) T(f)$, so if $T(f)$ is an epimorphism, $T(g)=T(h)$. Thus if $A^{\prime} \in \mathscr{R} \mathscr{F}(T)$, we must have $g=h$, and this shows that $f$ is an epimorphism.

(ii) This follows immediately from the definition of $\mathscr{R} \mathscr{F}(T)$.

Recall that a covariant functor $T: \mathscr{A} \rightarrow \mathscr{B}$ is said to reflect a property of a diagram in $\mathscr{A}$ if the condition that $T(D)$ has the property implies that $D$ has the property. Proposition 1.3 shows in particular that $T$ reflects epimorphisms and commutative diagrams in $\mathscr{R} \mathscr{F}(T)$.

If $A \in \mathscr{A}$, a family $\left\{U_{i}\right\}_{i \in I}$ of objects in $\mathscr{A}$ will be called a family of generators for $A$ if for all $X \in \mathscr{A}$ and $f, g \in \mathscr{A}(A, X), f \neq g$ implies there exists a morphism $u \in \mathscr{A}\left(U_{i}, A\right)$ for some $i \in I$ such that $f u \neq g u$. The family $\left\{U_{i}\right\}_{i \in I}$ is a family of generators for $\mathscr{A}$ if it is a family of generators for each $A \in \mathscr{A}$.

Following Lambek [4], a diagram $D$ in $\mathscr{A}$ is a functor $D: \mathscr{I} \rightarrow \mathscr{A}$, where $\mathscr{I}$ is a small category. An upper bound $(A, u)$ of $D$ consists of an object $A \in \mathscr{A}$ and a natural transformation $u: D \rightarrow A_{I}$, where $A_{I}: \mathscr{I} \rightarrow \mathscr{A}$ is the constant diagram which assigns $A$ to each object of $\mathscr{I}$ and $1_{A}$ to each morphism. If $(A, u)$ is an upper bound of $D$ such that for every upper bound $\left(A^{\prime}, u^{\prime}\right)$ of $D$ there exists a unique morphism $a: A \rightarrow A^{\prime}$ for which $a u(i)=u^{\prime}(i)$ for all objects $i \in \mathscr{I}$, then $(A, u)$ will be called a least upper bound or supremum of $D$, written $(A, u)=\sup (D)$, or simply $A=\sup (D)$. The greatest lower bound or infimum of $D$ is defined dually.

1.4. Proposition. Let $T: \mathscr{A} \rightarrow \mathscr{B}$ be a functor, and $A \in \mathscr{A}$. If $A$ has a family of generators in $\mathscr{R} \mathscr{F}(T)$, then $A \in \mathscr{R} \mathscr{F}(T)$.

Proof. Assume that $A$ has a family of generators $\left\{U_{i}\right\}_{i \in I}$ with $U_{i} \in \mathscr{R} \mathscr{F}(T)$ for each index $i \in I$. If $f, g \in \mathscr{A}(A, X)$ and $f \neq g$, then for some $i \in I$ there exists $u \in \mathscr{A}\left(U_{i}, A\right)$ with $f u \neq g u$. Since $U_{i} \in \mathscr{R} \mathscr{F}(T), T(f u) \neq T(g u)$ and consequently $T(f) \neq T(g)$, showing that $A \in \mathscr{R} \mathscr{F}(T)$.

1.5. Corollary. Let $T: \mathscr{A} \rightarrow \mathscr{B}$ be a functor. If $\mathscr{A}$ has a family of generators $\left\{U_{i}\right\}_{i \in I}$, then $T$ is faithful if and only if $U_{i} \in \mathscr{R} \mathscr{F}(T)$ for each $i \in I$.

1.6. CoRollary. If $D$ is a diagram in $\mathscr{R} \mathscr{F}(T)$, whose supremum exists in $\mathscr{A}$, then $\sup (D)=A \in \mathscr{R} \mathscr{F}(T)$.

Proof. Let $D: \mathscr{I} \rightarrow \mathscr{A}$ be a diagram with $D(i) \in \mathscr{R} \mathscr{F}(T)$ for each $i \in \mathscr{I}$, and which has a supremum $\sup (D)=(A, u)$ in $\mathscr{A}$. If $f, g \in \mathscr{A}(A, X)$, then $f u$ and $g u$ both define natural transformations $D \rightarrow X_{I}$, and the uniqueness properties for 
$(A, u)$ imply that if $f u_{i}=g u_{i}$ for all $u_{i}: D(i) \rightarrow A$, then $f=g$. This shows that the family $\{D(i)\}_{i \in \mathscr{I}}$ is a generating family for $A$, and so we must have $A \in \mathscr{R} \mathscr{F}(T)$.

Proposition 1.3 implies that if $f: A \rightarrow A^{\prime}$ is an epimorphism, then $A \in \mathscr{R} \mathscr{F}(T)$ implies $A^{\prime} \in \mathscr{R} \mathscr{F}(T)$. Corollary 1.6 shows that in particular $\mathscr{R} \mathscr{F}(T)$ is closed to the formation of direct sums (coproducts), cokernels, and pushouts, whenever they exist in $\mathscr{A}$.

If $\mathscr{A}$ is a sup-complete category, that is, if every diagram in $\mathscr{A}$ has a supremum, and $\mathscr{A}^{\prime}$ is a subcategory of $\mathscr{A}$, Corollary 1.6 makes it possible to define a supcompletion of $\mathscr{A}^{\prime}$ in $\mathscr{A}$ with respect to any collection of functors. Let $\mathscr{T}$ be a collection of functors, and let $\mathscr{A}^{\prime \prime}$ be the full subcategory defined by all $A \in \mathscr{A}$ such that $A \in \mathscr{R} \mathscr{F}(T)$ for all $T \in \mathscr{T}$ which act faithfully on the right of $\mathscr{A}^{\prime}$. It is clear that $\mathscr{A}^{\prime \prime}$ is sup-complete and that it contains all objects of $\mathscr{A}^{\prime}$.

The definition of $\mathscr{R} \mathscr{F}(T)$ can be generalized in the following way. If $\mathscr{A}^{\prime}$ is a class of objects of $\mathscr{A}$, let $\mathscr{R} \mathscr{F}\left(T, \mathscr{A}^{\prime}\right)$ be the full subcategory of $\mathscr{A}$ defined by all objects $A \in \mathscr{A}$ such that for all $A^{\prime} \in \mathscr{A}^{\prime}$ and $f, g \in \mathscr{A}\left(A, A^{\prime}\right), f \neq g$ implies $T(f)$ $\neq T(g)$. Thus $\mathscr{R} \mathscr{F}\left(T, \mathscr{A}^{\prime}\right)$ measures how faithfully $T$ acts on morphisms into objects of $\mathscr{A}^{\prime}$. Proposition 1.3 can be generalized to this situation.

A covariant functor $S: \mathscr{B} \rightarrow \mathscr{A}$ is said to be a left adjoint for a covariant functor $T: \mathscr{A} \rightarrow \mathscr{B}$ if there exists a natural equivalence of set-valued bifunctors $\eta: \mathscr{A}(S(-),-) \rightarrow \mathscr{B}(-, T(-))$, that is, for all $A \in \mathscr{A}, B \in \mathscr{B}$, we have $\eta_{B, A}: \mathscr{A}(S(B), A) \rightarrow \mathscr{B}(B, T(A))$. In particular, we have

$$
\eta_{T(A), A}: \mathscr{A}(S T(A), A) \rightarrow \mathscr{B}(T(A), T(A)),
$$

and we will denote by $\psi_{A}$ the inverse image of $1_{T(A)}$, so that $\psi_{A}: S T(A) \rightarrow A$. For each $B \in \mathscr{B}$ we define $\phi_{B}: B \rightarrow T S(B)$ dually.

1.7. Proposition. Let $T: \mathscr{A} \rightarrow \mathscr{B}$ and $S: \mathscr{B} \rightarrow \mathscr{A}$ be covariant functors with $S$ a left adjoint for $T$. Then the following are equivalent:

(i) $A \in \mathscr{R} \mathscr{F}(T)$.

(ii) For any $A^{\prime} \in \mathscr{A}$, if $f: A^{\prime} \rightarrow A$ and $T(f)$ is an epimorphism, then $f$ is an epimorphism.

(iii) For any $B \in \mathscr{B}$, if $f: B \rightarrow T(A)$ is an epimorphism, then $\eta^{-1}(f)$ is an epimorphism.

(iv) $\psi_{A}: S T(A) \rightarrow A$ is an epimorphism.

Proof. (i) $\Rightarrow$ (ii). This follows from Proposition 1.3.

(ii) $\Rightarrow$ (iii). If $f: B \rightarrow T(A)$ is an epimorphism, $\eta^{-1}(f): S(B) \rightarrow A$, and the diagram

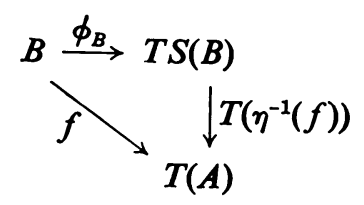


commutes. Thus if $f$ is an epimorphism, so is $T\left(\eta^{-1}(f)\right)$, and by assumption $\eta^{-1}(f)$ is an epimorphism.

(iii) $\Rightarrow$ (iv). This follows by taking $f=1_{T(A)}$.

(iv) $\Rightarrow$ (i). A morphism $f: A \rightarrow X$ induces the commutative diagram

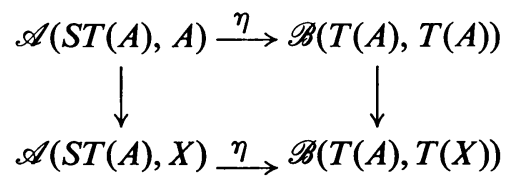

where $\eta\left(f \psi_{A}\right)=T(f) 1_{T(A)}=T(f)$. Then if $\psi_{A}$ is an epimorphism and $f, g: A \rightarrow X$, with $f \neq g$, we must have $f \psi_{A} \neq g \psi_{A}, \eta\left(f \psi_{A}\right) \neq \eta\left(g \psi_{A}\right)$, and therefore $T(f) \neq T(g)$. This shows that $A \in \mathscr{R} \mathscr{F}(T)$, and the proof is complete.

This generalizes Proposition 1.2, p. 119, of [6]. Note that in particular for all $B \in \mathscr{B}, S(B) \in \mathscr{R} \mathscr{F}(T)$.

If $T: \mathscr{A} \rightarrow \mathscr{B}$ and $S: \mathscr{B} \rightarrow \mathscr{A}$ are contravariant functors, they are said to be adjoint on the right if there exists a natural equivalence of set-valued bifunctors with $\eta_{A, B}: \mathscr{A}(A, S(B)) \rightarrow \mathscr{B}(B, T(A))$, for all $A \in \mathscr{A}$ and $B \in \mathscr{B}$. If we let $\psi_{A}$ $=\eta_{A, T(A)}^{-1}\left(1_{T(A)}\right)$, we have $\psi_{A}: A \rightarrow S T(A)$, as before. The proof of the following proposition is dual to that of Proposition 1.7.

1.8. Proposition. Let $T: \mathscr{A} \rightarrow \mathscr{B}$ and $S: \mathscr{B} \rightarrow \mathscr{A}$ be contravariant functors adjoint on the right. The following are equivalent:

(i) $A \in \mathscr{L} \mathscr{F}(T)$.

(ii) For any $A^{\prime} \in \mathscr{A}$, if $f: A \rightarrow A^{\prime}$ and $T(f)$ is an epimorphism, then $f$ is a monomorphism.

(iii) For any $B \in \mathscr{B}$, if $f: B \rightarrow T(A)$ is an epimorphism, then $\eta^{-1}(f)$ is a monomorphism.

(iv) $\psi_{A}: A \rightarrow S T(A)$ is a monomorphism.

2. In abelian categories. From this point on we restrict the categories studied to abelian categories $\mathscr{A}$ such that each $A \in \mathscr{A}$ has a representative class of subobjects which is a set, and a representative class of quotient objects which is a set. (That is, we assume that $\mathscr{A}$ is locally and colocally small.)

Using the terminology of Maranda [5], a subfunctor $T: \mathscr{A} \rightarrow \mathscr{A}$ of the identity is called a preradical. If $T^{2}=T$ it is called an idempotent preradical. If for all $A \in \mathscr{A}$ we have $T(K)=0$, where $K$ is the cokernel of the inclusion $a: T(A) \rightarrow A$, then $T$ is called a radical. If $T$ is a preradical, then for any morphism $f: A \rightarrow A^{\prime}$, $T(f)$ is the composition $f a$, where $a: T(A) \rightarrow A$ is the inclusion, and it is immediate that $A \in \mathscr{R} \mathscr{F}(T)$ if and only if $a: T(A) \rightarrow A$ is an isomorphism.

If $\mathscr{A}$ is sup-complete, and $\mathscr{A}^{\prime}=\mathscr{R} \mathscr{F}(T)$ for any functor $T: \mathscr{A} \rightarrow \mathscr{B}$, then $\mathscr{A}^{\prime}$ is also sup-complete. For each $A \in \mathscr{A}$ there is a representative set of subobjects of $A$ which belong to $\mathscr{A}^{\prime}$. Let $T^{\prime}(A)$ be the sum of these subobjects (the image of the obvious morphism from the direct sum of these objects into $A$ ). For $f: A \rightarrow X$, 
let $T^{\prime}(f)$ be the restriction of $f$ to $T^{\prime}(A)$. With this definition $T^{\prime}$ is an idempotent preradical and $A \in \mathscr{R} \mathscr{F}(T) \Leftrightarrow T^{\prime}(A)=A \Leftrightarrow A \in \mathscr{R} \mathscr{F}\left(T^{\prime}\right)$. In fact, we have shown the following.

2.1. Proposition. If $\mathscr{A}$ is sup-complete and $\mathscr{A}^{\prime}$ is a full subcategory of $A$, then the following are equivalent:

(i) $\mathscr{A}^{\prime}=\mathscr{R} \mathscr{F}(T)$ for a functor $T: \mathscr{A} \rightarrow \mathscr{B}$.

(ii) $\mathscr{A}^{\prime}$ is sup-complete.

(iii) $\mathscr{A}^{\prime}=\mathscr{R} \mathscr{F}(T)$ for an idempotent preradical $T: \mathscr{A} \rightarrow \mathscr{A}$.

If $T: \mathscr{A} \rightarrow \mathscr{B}$ is covariant and has a left adjoint $S: \mathscr{B} \rightarrow \mathscr{A}$, then for each $A \in \mathscr{A}$ let $T^{\prime}(A)$ be the image of $\psi_{A}: S T(A) \rightarrow A$. Since $S T(A) \in \mathscr{R} \mathscr{F}(T)$, we must have $T^{\prime}(A) \in \mathscr{R} \mathscr{F}(T)$. A morphism $f: A^{\prime} \rightarrow A$ induces a commutative diagram

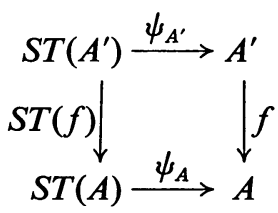

If $A^{\prime} \in \mathscr{R} \mathscr{F}(T)$, then $\psi_{A^{\prime}}$ is an epimorphism, which shows that the image of $f$ is contained in the image of $\psi_{A}$, that is, the image of $f$ is contained in $T^{\prime}(A)$. This implies that $T^{\prime}(A)$ is the largest subobject of $A$ which belongs to $\mathscr{R} \mathscr{F}(T)$, and $T^{\prime}$ in fact defines an idempotent preradical.

If $T$ is a radical, we may define an associated functor $1 / T: \mathscr{A} \rightarrow \mathscr{A}$ in the following way. For each $A \in \mathscr{A}$ let $(1 / T)(A)$ be the cokernel of the inclusion $T(A) \rightarrow A$. For $f: A \rightarrow A^{\prime}$, since $T$ is a preradical, $f(T(A)) \subseteq T\left(A^{\prime}\right)$, so $f$ induces a morphism $(1 / T)(f):(1 / T)(A) \rightarrow(1 / T)\left(A^{\prime}\right)$.

2.2. Proposition. If $\mathscr{A}$ is inf-complete and $\mathscr{A}^{\prime}$ is a full subcategory of $\mathscr{A}$, then the following are equivalent:

(i) $\mathscr{A}^{\prime}=\mathscr{L} \mathscr{F}(T)$ for a functor $T: \mathscr{A} \rightarrow \mathscr{B}$.

(ii) $\mathscr{A}^{\prime}$ is inf-complete.

(iii) $\mathscr{A}^{\prime}=\mathscr{L} \mathscr{F}(1 / T)$ for a radical $T: \mathscr{A} \rightarrow \mathscr{A}$, and $A \in \mathscr{A}^{\prime} \Leftrightarrow T(A)=0$.

Proof. We prove only that (ii) implies (iii). If $\mathscr{A}^{\prime}$ is inf-complete, let $T(A)$ be the intersection of the kernels of all homomorphisms from $A$ into objects of $\mathscr{A}^{\prime}$. With this definition, $T$ can be seen to determine a radical. Furthermore, $A \in \mathscr{A}^{\prime} \Leftrightarrow T(A)$ $=0$, and it is immediate that $A \in \mathscr{L} \mathscr{F}(1 / T) \Leftrightarrow T(A)=0$.

We now assume that all functors are additive. For an additive functor $T: \mathscr{A} \rightarrow \mathscr{B}, \mathscr{R} \mathscr{F}(T)$ is the full subcategory of all $A \in \mathscr{A}$ such that $0 \neq f: A \rightarrow X$ implies $T(f) \neq 0$. We next give some characterizations of $\mathscr{R} \mathscr{F}(T)$ when $T$ satisfies additional properties. We will call a functor a monofunctor if it is covariant and takes monomorphisms to monomorphisms or if it is contravariant and takes monomorphisms to epimorphisms. Epifunctors are defined dually. 
2.3. Proposition. Let $T: \mathscr{A} \rightarrow \mathscr{B}$ be an additive functor.

(i) If $T$ is a monofunctor, then $A \in \mathscr{R} \mathscr{F}(T) \Leftrightarrow T(f) \neq 0$ for all nonzero epimorphisms $f: A \rightarrow A^{\prime}$.

(ii) If $T$ is also an epifunctor, then $A \in \mathscr{R} \mathscr{F}(T) \Leftrightarrow T\left(A^{\prime}\right) \neq 0$ for all nonzero quotients $A^{\prime}$ of $A$.

Proof. (i) If $A \in \mathscr{R} \mathscr{F}(T)$, it must clearly satisfy the condition given in (i). On the other hand, suppose that $T$ is a monofunctor and $T(f) \neq 0$ for all nonzero epimorphisms $f: A \rightarrow A^{\prime}$. If $f: A \rightarrow X$ is any morphism, $f=f^{\prime} f^{\prime \prime}$ where $f^{\prime \prime}$ is an epimorphism and $f^{\prime}$ is a monomorphism. If $f \neq 0$, then $f^{\prime \prime} \neq 0$, so we must have $T\left(f^{\prime \prime}\right) \neq 0$. Since $f^{\prime}$ is monic and $T$ is a monofunctor, we must have $T\left(f^{\prime} f^{\prime \prime}\right) \neq 0$ and therefore $T(f) \neq 0$.

(ii) If $A \in \mathscr{R} \mathscr{F}(T)$, and $A^{\prime}$ is a nonzero quotient of $A$, then there is a nonzero epimorphism $f: A \rightarrow A^{\prime}$, and we must have $T(f) \neq 0$, so we cannot have $T\left(A^{\prime}\right)=0$. On the other hand, if $T\left(A^{\prime}\right) \neq 0$ for all nonzero quotients $A^{\prime}$ of $A$, then given $0 \neq f: A \rightarrow A^{\prime}$ with $f$ an epimorphism, we must have $T\left(A^{\prime}\right) \neq 0$ by assumption. Since $T$ is an epifunctor, this implies that $T(f) \neq 0$, and by (i) this shows that $A \in \mathscr{R} \mathscr{F}(T)$.

2.4. Proposition. Let $T: \mathscr{A} \rightarrow \mathscr{B}$ be an epifunctor. Let $0 \rightarrow A^{\prime} \stackrel{f}{\rightarrow} A \stackrel{g}{\rightarrow} A^{\prime \prime} \rightarrow 0$ be an exact sequence. If $A^{\prime}$ and $A^{\prime \prime} \in \mathscr{R} \mathscr{F}(T)$, then $A \in \mathscr{R} \mathscr{F}(T)$.

Proof. Let $h: A \rightarrow X$, with $h \neq 0$. If $h f \neq 0$, then since $A^{\prime} \in \mathscr{R} \mathscr{F}(T)$, we must have $T(h f) \neq 0$, and therefore $T(h) \neq 0$. On the other hand, if $h f=0$, then $h$ factors through $A^{\prime \prime}$, say $h=h^{\prime} g$, and $h \neq 0$ implies $h^{\prime} \neq 0$. Since $A^{\prime \prime} \in \mathscr{L} \mathscr{F}(T)$, this implies that $T\left(h^{\prime}\right) \neq 0$, and then $T(h)=T\left(h^{\prime} g\right) \neq 0$ because $T$ is an epifunctor. (If $T$ is covariant, $T(h)=T\left(h^{\prime}\right) T(g)$ and by assumption $T(g)$ is an epimorphism, so $T\left(h^{\prime}\right) T(g) \neq 0$. If $T$ is contravariant, $T(h)=T(g) T\left(h^{\prime}\right)$, where by assumption $T(g)$ is a monomorphism, and the desired conclusion again follows.)

If $A^{\prime}$ is a subobject of $A$, then $A$ is said to be an essential extension of $A^{\prime}$ if $A^{\prime} \cap A^{\prime \prime} \neq 0$ for all nonzero subjects $A^{\prime \prime}$ of $A$. The intersection of two subobjects is the pullback of the inclusion maps, so that $A^{\prime} \rightarrow A$ is an essential monomorphism if and only if for all nonzero subobjects $A^{\prime \prime} \rightarrow A$ we have $P \rightarrow A^{\prime} \neq 0$ in the pullback diagram

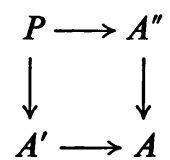

It can be shown that a monomorphism $A^{\prime} \rightarrow A$ is essential if and only if $P \rightarrow A^{\prime}$ $\neq 0$ for the pullback of any nonzero morphism $f: X \rightarrow A$, where we have

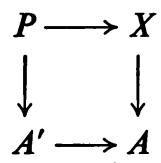


This characterization can be used to prove the following proposition.

2.5. Proposition. Let $T: \mathscr{A} \rightarrow \mathscr{B}$ be a monofunctor. If $A^{\prime} \in \mathscr{L} \mathscr{F}(T)$, and $A^{\prime} \rightarrow A$ is an essential monomorphism, then $A \in \mathscr{L} \mathscr{F}(T)$.

Proof. Let $0 \neq f: X \rightarrow A$. Constructing the pullback diagram gives

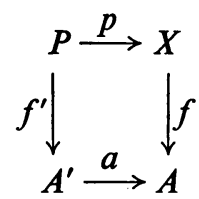

If $f \neq 0$ and $A^{\prime} \rightarrow A$ is essential, then $f^{\prime} \neq 0$.

Since $A^{\prime} \in \mathscr{L} \mathscr{F}(T)$, we must have $T\left(f^{\prime}\right) \neq 0$, and then because $T$ is a monofunctor, we must have $T\left(a f^{\prime}\right) \neq 0$. Therefore $T(f p) \neq 0$, which forces $T(f) \neq 0$.

For a functor $T: \mathscr{A} \rightarrow \mathscr{B}$ define $\mathscr{K}_{e r}(T)$ as the full subcategory determined by all $A \in \mathscr{A}$ such that $T(A)=0$. If $A \in \mathscr{R} \mathscr{F}(T)$ and $A^{\prime} \in \mathscr{K} e r(T)$, then if $f: A \rightarrow A^{\prime}$, we must have $T(f)=0$ because $T\left(A^{\prime}\right)=0$. Since $A \in \mathscr{R} \mathscr{F}(T)$, this implies that $f=0$, and we see that $\mathscr{A}\left(A, A^{\prime}\right)=0$.

2.6. Proposition. Let $T: \mathscr{A} \rightarrow \mathscr{B}$ be an exact functor. Then

(i) $A \in \mathscr{R} \mathscr{F}(T) \Leftrightarrow \mathscr{A}(A, K)=0$ for all $K \in \mathscr{K}_{e r}(T)$.

(ii) $A \in \mathscr{L} \mathscr{F}(T) \Leftrightarrow \mathscr{A}(K, A)=0$ for all $K \in \mathscr{K}_{e r}(T)$.

Proof. (i) If $A \in \mathscr{R} \mathscr{F}(T)$, then $\mathscr{A}(A, K)=0$ for all $K \in \mathscr{K} e r(T)$. On the other hand, if $\mathscr{A}(A, K)=0$ for all $K \in \mathscr{K}_{e r}(T)$, then suppose that $f: A \rightarrow A^{\prime}$ is a nonzero epimorphism. Since $\mathscr{A}\left(A, A^{\prime}\right) \neq 0$ we must have $T\left(A^{\prime}\right) \neq 0$. Because $T$ is exact, $T\left(A^{\prime}\right) \neq 0$ implies $T(f) \neq 0$, and $A \in \mathscr{R} \mathscr{F}(T)$ by Proposition 2.3.

(ii) This is proved similarly, using the dual of Proposition 2.3.

2.7. Proposition. Let $T: \mathscr{A} \rightarrow \mathscr{B}$ be covariant, and assume that $T$ has a left adjoint $S: \mathscr{B} \rightarrow \mathscr{A}$. Then $K \in \mathscr{K}$ er $(T) \Leftrightarrow \mathscr{A}(A, K)=0$ for all $A \in \mathscr{R} \mathscr{F}(T)$.

Proof. If $K \in \mathscr{K}_{e r}(T)$, then by previous results $\mathscr{A}(A, K)=0$ for all $A \in \mathscr{R} \mathscr{F}(T)$. On the other hand, by Proposition 1.7, $S T(A) \in \mathscr{R} \mathscr{F}(T)$ for all $A \in \mathscr{A}$, so if $\mathscr{A}(A, K)=0$ for all $A \in \mathscr{R} \mathscr{F}(T)$, then in particular, $\mathscr{A}(S T(K), K)=0$, so $\psi_{K}=0$, where $\psi_{K}: S T(K) \rightarrow K$ is the inverse image of $1_{T(K)}$ under the natural isomorphism $\eta$ which defines the adjoint situation. Because $\eta$ is additive, we must have $1_{T(K)}=0$, which shows that $K \in \mathscr{K}_{e r}(T)$.

2.8. Proposition. Let $T: \mathscr{A} \rightarrow \mathscr{B}$ be an exact, covariant functor, and assume that $T$ has a left adjoint $S: \mathscr{B} \rightarrow \mathscr{A}$. For each $A \in \mathscr{A}$ let $r(A)$ denote the maximal subobject of $A$ which belongs to $\mathscr{R} \mathscr{F}(T)$. Then for each $A \in \mathscr{A}, T(r(A)) \approx T(A)$.

Proof. Each $A \in \mathscr{A}$ does possess a maximal subobject belonging to $\mathscr{R} \mathscr{F}(T)$, namely the image of $\psi_{A}: S T(A) \rightarrow A$. Let $K$ be the cokernel of the inclusion map $r(A) \rightarrow A$. This gives an exact sequence $0 \rightarrow r(A) \rightarrow A \rightarrow K \rightarrow 0$, and since $T$ is exact, we have the exact sequence $0 \rightarrow T(r(A)) \rightarrow T(A) \rightarrow T(K) \rightarrow 0$. To show that $T(r(A)) \approx T(A)$, it thus suffices to show that $T(K)=0$, and to do this we apply 
Proposition 2.7. We claim that $\mathscr{A}(A, K)=0$ for all $A \in \mathscr{R} \mathscr{F}(T)$. If not, then since $\mathscr{R} \mathscr{F}(T)$ is closed to formation of quotients, there exists a subobject $0 \neq K^{\prime} \subseteq K$ such that $K^{\prime} \in \mathscr{R} \mathscr{F}(T)$, and if $A^{\prime}$ is the inverse image in $A$ of $K^{\prime}$, we have an exact sequence $0 \rightarrow r(A) \rightarrow A^{\prime} \rightarrow K^{\prime} \rightarrow 0$. By Proposition 2.4 and the exactness of $T$, this implies that $A^{\prime} \in \mathscr{R} \mathscr{F}(T)$, a contradiction, since $r(A) \varsubsetneqq A^{\prime}$ and $r(A)$ is the largest subobject of $A$ belonging to $\mathscr{R} \mathscr{F}(T)$.

3. Generating and cogenerating structures. Recall that $U \in \mathscr{A}$ is called a generator for $\mathscr{A}$ if the functor $H^{U}: \mathscr{A} \rightarrow \mathscr{E}$ ns is faithful, where $H^{U}(A)$ $=\operatorname{Hom}_{\mathscr{A}}(U, A)=\mathscr{A}(U, A)$. Since $\mathscr{A}$ is abelian, $U$ is a generator for $\mathscr{A}$ if and only if for each $A \in \mathscr{A}$ and $0 \neq f: A \rightarrow X$ there exists $g: U \rightarrow A$ with $f g \neq 0$. If $H^{U}$ is not faithful, then we may consider $\mathscr{R} \mathscr{F}\left(H^{U}\right)$.

3.1. Definition. Let $U, A \in \mathscr{A}$. If for each morphism $0 \neq f: A \rightarrow X$ in $\mathscr{A}$ there exists a morphism $g: U \rightarrow A$ in $\mathscr{A}$ with $f g \neq 0$, we will write $U \succ A$.

From the definition we see that if $A \succ A^{\prime}$ and $A^{\prime} \succ A^{\prime \prime}$, then $A \succ A^{\prime \prime}$. Moreover, $U \succ A$ if and only if $A \in \mathscr{R} \mathscr{F}\left(H^{U}\right)$. If $\mathscr{A}$ is sup-complete, then $H^{U}$ has a left adjoint $S: \mathscr{E} n s \rightarrow \mathscr{A}$, where $S(I)=U^{I}$ for any set $I \in \mathscr{E} n s$, and $S$ acts in the obvious way on functions. (Here we use $U^{I}$ to denote the direct sum of $I$ copies of $U$.) As before, for each $A \in \mathscr{A}$, the adjoint situation gives rise to $\psi_{A}: U^{I} \rightarrow A$, where $I=\mathscr{A}(U, A)$. We will let $\operatorname{tr}_{A}(U)$ denote the image of $\psi_{A}$. Proposition 1.7 can be used to show that the following are equivalent: (i) $A \in \mathscr{R} \mathscr{F}\left(H^{U}\right)$, (ii) $U>A$, (iii) $\operatorname{tr}_{A}(U)=A$, (iv) $\psi_{A}: U^{I} \rightarrow A$ is an epimorphism, where $I=\mathscr{A}(U, A)$. The equivalence of (ii), (iii), and (iv) is well known.

If $\mathscr{A}$ is sup-complete and $\mathscr{A}^{\prime}$ is a nonempty class of objects of $\mathscr{A}$, we can define a sup-completion of $\mathscr{A}^{\prime}$ with respect to all covariant Hom-functors, by letting $\mathscr{A}^{\prime \prime}$ be the full subcategory defined by all objects $A \in \mathscr{A}$ such that $A \in \mathscr{R} \mathscr{F}\left(H^{U}\right)$ for all $U \in \mathscr{A}$ such that $\mathscr{R} \mathscr{F}\left(H^{U}\right) \supseteq \mathscr{A}^{\prime}$. This gives rise to what we will call a generating structure.

3.2. Definition. For a nonempty class $\mathscr{A}^{\prime}$ of objects of $\mathscr{A}$, let $\Phi\left(\mathscr{A}^{\prime}\right)$ denote the class of all $A \in \mathscr{A}$ such that $A>A^{\prime}$ for all $A^{\prime} \in \mathscr{A}^{\prime}$, and let $\Psi\left(\mathscr{A}^{\prime}\right)$ denote the class of all $A \in \mathscr{A}$ such that $A^{\prime}>A$ for all $A^{\prime} \in \mathscr{A}^{\prime}$.

3.3. Definition. If $\mathscr{C}$ and $\mathscr{D}$ are nonempty classes of objects of $\mathscr{A}$, then the pair $(\mathscr{C}, \mathscr{D})$ is called a generating structure if $\mathscr{C}=\Phi(\mathscr{D})$ and $\mathscr{D}=\Psi(\mathscr{C})$. If $\mathscr{C}=\Phi(A)$ and $\mathscr{D}=\Psi(A)$ for some $A \in \mathscr{A}$, we say that $(\mathscr{C}, \mathscr{D})$ is supported by $A$.

It is immediate from the definition that $A$ is a support for the generating structure $(\mathscr{C}, \mathscr{D})$ if and only if $A \in \mathscr{C} \cap \mathscr{D}$. Since $\mathscr{D}$ is closed to the formation of quotient objects, $A$ is a generator for the full subcategory determined by $\mathscr{D}$ if and only if $(\mathscr{C}, \mathscr{D})$ is supported by $A$.

If $\mathscr{A}$ is sup-complete, then any generating structure $(\mathscr{C}, \mathscr{D})$ such that $\mathscr{D}$ is closed to formation of subobjects has a supporting object. To show this, let $U$ be any object of $\mathscr{C}$ and let $A$ be the direct sum of a representative set of quotients of $U$ which belong to $\mathscr{D}$. Then $A \in \mathscr{D}$ because $\mathscr{D}$ is sup-complete. If $D \in \mathscr{D}$ and 
$0 \neq f: D \rightarrow X$, then since $U \in \mathscr{C}$ there exists $g: U \rightarrow D$ such that $f g \neq 0$, and because $\mathscr{D}$ is closed to formation of subobjects, $\operatorname{Im}(g) \in \mathscr{D}$. From the definition of $A$ there exists an epimorphism $h: A \rightarrow \operatorname{Im}(g)$, so if $i$ is the inclusion $i: \operatorname{Im}(g) \rightarrow D$, then fih $\neq 0$ and this shows that $A \succ D$. Therefore $A \in \mathscr{C}$ and $(\mathscr{C}, \mathscr{D})$ is supported by $A$.

The next proposition gives several methods of constructing generating structures.

3.4. Proposition. Let $\mathscr{A}^{\prime}$ be a class of $\mathscr{A}$, and let $A \in \mathscr{A}^{\prime}$. If $\Phi\left(\mathscr{A}^{\prime}\right)$ is nonempty, then each of the following is a generating structure:

(i) $\left(\Phi\left(\mathscr{A}^{\prime}\right), \Psi \Phi\left(\mathscr{A}^{\prime}\right)\right)$,

(ii) $\left(\Phi \Psi\left(\mathscr{A}^{\prime}\right), \Psi\left(\mathscr{A}^{\prime}\right)\right)$,

(iii) $(\Phi(A), \Psi(A))$.

Proof. (i) and (ii) follow directly from the definitions and the fact that $\Phi \Psi \Phi=\Phi$ and $\Psi \Phi \Psi=\Psi$.

(iii) follows from the transitivity of $>$ and the fact that $A \in \Phi(A)$ and $A \in \Psi(A)$.

We next give an elementary result which compares generating structures. It will be very useful in the next section.

3.5. Proposition. Let $(\mathscr{C}, \mathscr{D})$ and $\left(\mathscr{C}^{\prime}, \mathscr{D}^{\prime}\right)$ be generating structures supported by $U$ and $U^{\prime}$ respectively. Then the following are equivalent:

(i) $\mathscr{C} \subseteq \mathscr{C}^{\prime}$,

(ii) $U \in \mathscr{C}^{\prime}$,

(iii) $\mathscr{D} \supseteq \mathscr{D}^{\prime}$,

(iv) $U^{\prime} \in \mathscr{D}$.

Proof. (i) $\Rightarrow$ (ii). Since $U \in \mathscr{C}$ and $\mathscr{C} \subseteq \mathscr{C}^{\prime}$, we have $U \in \mathscr{C}^{\prime}$.

(ii) $\Rightarrow$ (iii). If $U \in \mathscr{C}^{\prime}$, then $U \succ D^{\prime}$ for all $D^{\prime} \in \mathscr{D}^{\prime}$, and therefore $\mathscr{D}^{\prime} \subseteq \Psi(U)=\mathscr{D}$.

(iii) $\Rightarrow$ (iv). Since $U^{\prime} \in \mathscr{D}^{\prime}$ and $\mathscr{D}^{\prime} \subseteq \mathscr{D}$, we have $U^{\prime} \in \mathscr{D}$.

(iv) $\Rightarrow$ (i). If $U^{\prime} \in \mathscr{D}$, then $C \succ U^{\prime}$ for all $C \in \mathscr{C}$, and therefore $\mathscr{C} \subseteq \Phi\left(U^{\prime}\right)=\mathscr{C}^{\prime}$.

We now give some dual definitions. Recall that $Q \in \mathscr{A}$ is called a cogenerator for $\mathscr{A}$ if the contravariant functor $H_{Q}: \mathscr{A} \rightarrow \mathscr{E} n s$ is faithful, where $H_{Q}(A)=\mathscr{A}(A, Q)$ for all $A \in \mathscr{A}$. Equivalently, for each morphism $0 \neq f: X \rightarrow A$ there exists $g: A \rightarrow Q$ with $f g \neq 0$. This motivates the following definition.

3.6. Definition. Let $A, Q \in \mathscr{A}$. Then $A>Q$ if for each morphism $0 \neq f: X \rightarrow A$ in $\mathscr{A}$ there exists a morphism $g: A \rightarrow Q$ in $\mathscr{A}$ such that $g f \neq 0$.

Again it is clear that if $A>A^{\prime}$ and $A^{\prime}>A^{\prime \prime}$, then $A>A^{\prime \prime}$, and that $A>Q$ if and only if $A \in \mathscr{L} \mathscr{F}\left(H_{Q}\right)$. If $\mathscr{A}$ is inf-complete, then $H_{Q}$ has an adjoint on the right, given by $S: \mathscr{E} n s \rightarrow \mathscr{A}$ where $S(I)=Q^{(I)}$ is the product of $I$ copies of $Q$. The adjoint situation induces for each $A \in \mathscr{A}$ a morphism $\psi_{A}: A \rightarrow Q^{(I)}$, where $I=\mathscr{A}(A, Q)$. Proposition 1.8 shows that the following are equivalent: (i) $A \in \mathscr{L} \mathscr{F}\left(H_{Q}\right)$, (ii) $A>Q$, (iii) $\psi_{A}: A \rightarrow Q^{(I)}, I=\mathscr{A}(A, Q)$, is a monomorphism.

3.7. Definition. Let $\mathscr{A}^{\prime}$ be a nonempty class of objects of $\mathscr{A}$. Let $\Phi^{*}\left(\mathscr{A}^{\prime}\right)$ denote the class of all $A \in \mathscr{A}$ such that $A^{\prime}>A$ for all $A^{\prime} \in \mathscr{A}^{\prime}$. Let $\Psi^{*}\left(\mathscr{A}^{\prime}\right)$ denote the class of all $A \in \mathscr{A}$ such that $A>A^{\prime}$ for all $A^{\prime} \in \mathscr{A}^{\prime}$. 
3.8. Definition. If $\mathscr{C}$ and $\mathscr{D}$ are nonempty classes of objects of $\mathscr{A}$, then the pair $(\mathscr{C}, \mathscr{D})$ is called a cogenerating structure if $\mathscr{C}=\Phi^{*}(\mathscr{D})$ and $\mathscr{D}=\Psi^{*}(\mathscr{C})$. If $\mathscr{C}=\Phi^{*}(A)$ and $\mathscr{D}=\Psi^{*}(A)$ for some $A \in \mathscr{A}$, we say that $(\mathscr{C}, \mathscr{D})$ is supported by $A$.

The proof of the following proposition is dual to that of Proposition 3.4.

3.9. Proposition. Let $\mathscr{A}^{\prime}$ be a class of objects of $\mathscr{A}$, and let $A \in \mathscr{A}^{\prime}$. If $\Phi^{*}\left(\mathscr{A}^{\prime}\right)$ is nonempty, then each of the following is a cogenerating structure:

(i) $\left(\Phi^{*}\left(\mathscr{A}^{\prime}\right), \Psi^{*} \Phi^{*}\left(\mathscr{A}^{\prime}\right)\right)$,

(ii) $\left(\Phi^{*} \Psi^{*}\left(\mathscr{A}^{\prime}\right), \Psi^{*}\left(\mathscr{A}^{\prime}\right)\right)$,

(iii) $\left(\Phi^{*}(A), \Psi^{*}(A)\right)$.

If $\mathscr{A}$ is inf-complete, then as before it is possible to show that any cogenerating structure $(\mathscr{C}, \mathscr{D})$ such that $\mathscr{D}$ is closed to formation of quotients has a supporting object. If $\mathscr{A}$ has a generator and each object of $\mathscr{A}$ has an injective envelope, then any cogenerating structure $(\mathscr{C}, \mathscr{D})$ such that $\mathscr{D}$ is closed to essential extensions has a supporting object $A$. To show this, if $U$ is a generator for $\mathscr{A}$, let $A$ be the injective envelope of the product of a representative set of quotients of $U$ which belong to $\mathscr{D}$. If $D \in \mathscr{D}$ and $0 \neq f: X \rightarrow D$, then $\operatorname{Im}(f) \rightarrow D \neq 0$, so there exists $g: U \rightarrow \operatorname{Im}(f)$ such that $\operatorname{Im}(g) \neq 0$. Because $\mathscr{D}$ is closed to formation of subobjects, $\operatorname{Im}(g) \in \mathscr{D}$, and is isomorphic to a quotient of $U$. We therefore have a morphism $h: \operatorname{Im}(g) \rightarrow A$ which extends by the injectivity of $A$ to $h^{\prime}: D \rightarrow A$, and we have $h^{\prime} f \neq 0$. This shows that $D>A$ for all $D \in \mathscr{D}$, so $A \in \mathscr{C}$. Because $\mathscr{D}$ is inf-complete and closed to formation of essential extensions we also have $A \in \mathscr{D}$.

Generating and cogenerating structures appear in the study of torsion theories. A radical $T: \mathscr{A} \rightarrow \mathscr{A}$ is called a torsion radical if it is left exact. Let

$$
\mathscr{D}=\{A \in \mathscr{A}: T(A)=A\} \text { and } \mathscr{D}^{*}=\{A \in \mathscr{A}: T(A)=0\} .
$$

If $T$ is a torsion radical, then $\mathscr{D}$ is closed to formation of subobjects and $\mathscr{D}^{*}$ is closed to essential extensions. (See $[5, \S 6]$ and $[4, \S 10]$.) If $\mathscr{A}$ is sup-complete and inf-complete, then we know that $\mathscr{D}$ is sup-complete and $\mathscr{D}^{*}$ is inf-complete. If in addition $\mathscr{A}$ has a generator and injective envelopes for all objects, then there is an object $U$ such that $\mathscr{D}=\Psi(U)$ and an injective object $Q$ such that $\mathscr{D}^{*}=\Psi^{*}(Q)$. Thus $(\Phi(U), \mathscr{D})$ is a generating structure associated with $T$ and $\left(\Phi^{*}(Q), \mathscr{D}^{*}\right)$ is a cogenerating structure associated with $T$.

For completeness we state the dual of Proposition 3.5. From the remarks above, it could be used together with Proposition 3.5 to compare the classes of objects determined by two torsion theories.

3.10. Proposition. Let $(\mathscr{C}, \mathscr{D})$ and $\left(\mathscr{C}^{\prime}, \mathscr{D}^{\prime}\right)$ be cogenerating structures supported by $Q$ and $Q^{\prime}$ respectively. Then the following are equivalent:

(i) $\mathscr{C} \subseteq \mathscr{C}^{\prime}$,

(ii) $Q \in \mathscr{C}^{\prime}$,

(iii) $\mathscr{D} \supseteq \mathscr{D}^{\prime}$,

(iv) $Q^{\prime} \in \mathscr{D}$. 
3.11. Proposition. Let $\mathscr{A}$ be a sup-complete and inf-complete category, and let $A, U, Q$ be objects of $\mathscr{A}$.

(i) $A>Q \Leftrightarrow$ there exists a subobject $Q^{\prime} \subseteq Q$ such that $A>Q^{\prime}$ and $A>Q^{\prime}$.

(ii) $U \succ A \Leftrightarrow$ there exists a quotient $U^{\prime}$ of $U$ such that $U^{\prime}>A$ and $U^{\prime}>A$.

Proof. We prove only (i), since the proof of (ii) is dual. If $A>Q$, let $Q^{\prime}=\operatorname{tr}_{Q}(A)$. Clearly $A>\operatorname{tr}_{Q}(A)$, and since $\operatorname{Im}(f) \subseteq \operatorname{tr}_{Q}(A)$ for all $f: A \rightarrow Q$, it follows from the definition of $>$ that $A>Q$ implies $A>\operatorname{tr}_{Q}(A)$. Thus $A>Q^{\prime}$ and $A>Q^{\prime}$.

4. Generating and cogenerating structures in categories of modules. In this section we specialize to categories of modules. We will assume that all rings are associative rings with identity and that all modules are unital. To fix some notation, we let $R$ denote an associative ring with identity and ${ }_{R} \mathscr{M}$ denote the category of unital left $R$-modules. We will investigate generating and cogenerating structures supported by certain canonical $R$-modules, but before doing so we obtain some additional information about Hom-functors in this situation. If ${ }_{R} U_{S}$ is an $R-S$ bimodule, then $\operatorname{Hom}_{R}(U, M)$ is a left $S$-module, for any module ${ }_{R} M$. In this case ${ }_{S} \operatorname{Hom}_{R}(U,-):{ }_{R} \mathscr{M} \rightarrow{ }_{S} \mathscr{M}$ has a left adjoint ${ }_{R} U \otimes_{S}(-):{ }_{S} \mathscr{M} \rightarrow{ }_{R} \mathscr{M}$. Forgetting the $S$-module structure gives $\operatorname{Hom}_{R}(U,-):{ }_{R} \mathscr{M} \rightarrow \mathscr{E} n s$, and it is clear that $\mathscr{R} \mathscr{F}\left(\operatorname{Hom}_{R}(U,-)\right)=\mathscr{R} \mathscr{F}\left({ }_{S} \operatorname{Hom}_{R}(U,-)\right)$, so for ${ }_{R} M \in{ }_{R} \mathscr{M}$, the image of $\psi_{M}:{ }_{R} U \otimes_{S} \operatorname{Hom}_{R}(U, M) \rightarrow M$ given by $\sum_{i=1}^{n} u_{i} \otimes f_{i}=\sum_{i=1}^{n} f_{i}\left(u_{i}\right)$ is precisely $\operatorname{tr}_{M}(U)$, and $\psi_{M}$ is an epimorphism if and only if $U^{I} \rightarrow M$ is an epimorphism, where $I=\operatorname{Hom}_{R}(U, M)$.

Using the adjointness we can also describe $\mathscr{L} \mathscr{F}\left({ }_{R} U \otimes{ }_{S}(-)\right)$ by applying the dual of Proposition 1.7. The adjoint situation gives rise to

$$
\phi_{M}:{ }_{S} M \rightarrow{ }_{S} \operatorname{Hom}_{R}\left(U, U \otimes{ }_{S} M\right),
$$

defined by $\phi_{M}(m)=f_{m}$, where $f_{m}:{ }_{R} U \rightarrow{ }_{R} U \otimes{ }_{S} M$ and $f_{m}(u)=u \otimes m$, for all $u \in U$. $\phi_{M}$ is a monomorphism if and only if for each $0 \neq m \in M$ there exists $u \in U$ such that $u \otimes m \neq 0$ in $U \otimes{ }_{S} M$. The dual of Proposition 1.7 shows that the following are equivalent: (i) ${ }_{s} M \in \mathscr{L} \mathscr{F}\left({ }_{R} U \otimes{ }_{S}(-)\right)$. (ii) If $0 \neq f:{ }_{S} N \rightarrow{ }_{S} M$, then $U \otimes f:{ }_{R} U \otimes{ }_{S} N \rightarrow{ }_{R} U \otimes{ }_{S} M$ is also nonzero. (iii) If $f:{ }_{S} M \rightarrow{ }_{S} N$ and $U \otimes f:{ }_{R} U \otimes{ }_{S} M \rightarrow{ }_{R} U \otimes{ }_{S} N$ is a monomorphism, then $f$ is a monomorphism. (iv) For each $0 \neq m \in M$ there exists $u \in U$ such that $0 \neq u \otimes m \in{ }_{R} U \otimes{ }_{S} M$.

If ${ }_{R} Q_{S}$ is an $R$-S-bimodule, we can also give additional information regarding $\operatorname{Hom}_{R}(-, Q)$. For each ${ }_{R} M \in{ }_{R} \mathscr{M}, \operatorname{Hom}_{R}(M, Q)$ is a right $S$-module, and the functor ${ }_{R} \operatorname{Hom}_{S}(-, Q): \mathscr{M}_{S} \rightarrow{ }_{R} \mathscr{M}$ is adjoint on the right to $\operatorname{Hom}_{R}(-, Q)_{S}:{ }_{R} \mathscr{M} \rightarrow \mathscr{M}_{S}$. It is customary to let $\operatorname{Hom}_{R}(M, Q)_{S}=M^{*}$, and ${ }_{R} \operatorname{Hom}_{S}\left(M^{*}, Q\right)=M^{* *}$. The adjoint situation induces the morphism $\psi_{M}: M \rightarrow M^{* *}$ for all $M \in{ }_{R} \mathscr{M}$. It is clear that for $\operatorname{Hom}_{R}(-, Q):{ }_{R} \mathscr{M} \rightarrow \mathscr{E} n s$ we must have $\mathscr{L} \mathscr{F}\left(\operatorname{Hom}_{R}(-, Q)\right)$ $=\mathscr{L} \mathscr{F}\left(\operatorname{Hom}_{R}(-, Q)_{S}\right)$. Using this fact we can apply Proposition 1.8 to obtain the well-known equivalence of the following: (i) ${ }_{R} M>_{R} Q$. (ii) If $0 \neq f:{ }_{R} N \rightarrow{ }_{R} M$, then $f^{*}: M^{*} \rightarrow N^{*}$ is also nonzero. (iii) If $f:{ }_{R} M \rightarrow{ }_{R} N$ and $f^{*}: N^{*} \rightarrow M^{*}$ is an epi- 
morphism, then $f$ is a monomorphism. (iv) $\psi_{M}: M \rightarrow M^{* *}$ is a monomorphism. (v) $M \rightarrow Q^{(I)}$ is a monomorphism, where $I=\operatorname{Hom}_{R}(M, Q)$.

If $A$ is a left ideal of $R$ and $Q$ is a module, we define $r_{Q}(A)=\{q \in Q: A q=0\}$, and for any subset $Q^{\prime}$ of $Q$ we define $\ell_{R}\left(Q^{\prime}\right)=\left\{r \in R: r q^{\prime}=0\right.$ for all $\left.q^{\prime} \in Q^{\prime}\right\}$. In particular, we have $\ell_{R}(Q)=\operatorname{Ann}(Q)$.

4.1. Proposition. Let ${ }_{R} M,{ }_{R} Q \in{ }_{R} M$.

(i) Let $A$ be a two-sided ideal of $R$. Then $R / A>M \Leftrightarrow A \subseteq$ Ann (M).

(ii) If $M^{\prime}$ is a submodule of $M$, then $M / M^{\prime}>Q \Leftrightarrow$ for all $m \in M, m \notin M^{\prime}$ implies there exists $f:{ }_{R} M \rightarrow{ }_{R} Q$ with $f\left(M^{\prime}\right)=0$ and $f(m) \neq 0$.

(iii) Let $A$ be a left ideal of $R$. Then $R / A>Q \Leftrightarrow \ell_{R}\left(\imath_{Q}(A)\right)=A$.

Proof. (i) If $R / A \succ M$, then if $m \in M$ we must have $m=\sum_{i=1}^{n} f_{i}\left(r_{i}\right)$ for $f_{i} \in \operatorname{Hom}_{R}(R / A, M)$ and $r_{i} \in R$. But then for $a \in A$ we must have $a m=a\left(\sum_{i=1}^{n} f_{i}\left(r_{i}\right)\right)$ $=\sum_{i=1}^{n} f_{i}\left(a r_{i}\right)=0$, since $a r_{i} \in A$ for all $i$. This shows that $A \subseteq$ Ann $(M)$. Conversely, we know that $R>M$, and since Ann $(M)$ is the intersection of the kernels of all $R$-homomorphisms from $R$ to $M$, we have $R /$ Ann $(M) \succ M$ and $R /$ Ann $(M)>M$. (See Proposition 3.11.) Then if $A \subseteq$ Ann $(M), R / A \succ R /$ Ann $(M)$ and therefore $R / A>M$

(ii) If $M / M^{\prime}>Q$, then for $m \in M, m \notin M^{\prime}$, we must have $p(m) \neq 0$ for the projection $p: M \rightarrow M / M^{\prime}$. Since $M / M^{\prime}>Q$, there exists $f: M / M^{\prime} \rightarrow Q$ such that $f(p(m)) \neq 0$, so $f p: M \rightarrow Q$ and $f p\left(M^{\prime}\right)=0$ but $f p(m) \neq 0$. Conversely, if $m \in M$ and $p(m) \neq 0$, there exists $f: M \rightarrow Q$ with $f(m) \neq 0$ and $f\left(M^{\prime}\right)=0$. Since $f\left(M^{\prime}\right)=0, f$ factors through $p$, say $f=f^{\prime} p$, and then $f^{\prime}(p(m)) \neq 0$. This shows that $M / M^{\prime}>Q$.

(iii) We have $A \subseteq \ell_{R}\left(\imath_{Q}(A)\right)$ for all $Q$. If $R / A>Q$ and $r \in R$ but $r \notin A$, then by (ii) there exists $f: R \rightarrow Q$ with $f(r) \neq 0$ and $f(A)=0$. This implies that $A f(1)=0$ but $r f(1) \neq 0$, so $r \notin \ell_{R}\left(\iota_{Q}(A)\right)$. On the other hand, if $A=\ell_{R}\left(\iota_{Q}(A)\right)$ and $r \in R, r \notin A$, then $r \notin \ell_{R}\left(r_{Q}(A)\right)$ and therefore there exists $q \in Q$ such that $A q=0$ but $r q \notin 0$. Define $f: R \rightarrow Q$ by $f(1)=q$. Then $f(A)=0$ and $f(r) \neq 0$, so by (ii), $R / A>Q$.

4.2. Proposition. Let $(\mathscr{C}, \mathscr{D})$ be a cogenerating structure supported by ${ }_{R} Q$. If $E(Q)$ is the injective envelope of $Q$, then $\mathscr{D}$ is closed to formation of essential extensions $\Leftrightarrow(\mathscr{C}, \mathscr{D})$ is supported by $E(Q)$.

Proof. $(\Rightarrow)$. Since $Q>E(Q)$ and $Q \in \mathscr{C}$, we must have $E(Q) \in \mathscr{C}$. By assumption an essential extension of any module in $\mathscr{D}$ is also in $\mathscr{D}$, so since $Q \in \mathscr{D}$ and $E(Q)$ is essential over $Q$, we must have $E(Q) \in \mathscr{D}$. This shows that $(\mathscr{C}, \mathscr{D})$ is supported by $E(Q)$.

$(\Leftarrow)$. If $(\mathscr{C}, \mathscr{D})$ is supported by $E(Q)$, then $\mathscr{D}=\Psi^{*}(E(Q))=\mathscr{L} \mathscr{F}\left(H_{E(Q)}\right)$. The functor $H_{E(Q)}:{ }_{R} \mathscr{M} \rightarrow \mathscr{E} n s$ is a monofunctor since $E(Q)$ is injective. Proposition 2.5 then implies that $\mathscr{D}$ is closed to formation of essential extensions.

We will say that a class $\mathscr{A}$ of modules is categorically defined (or simply that it is categorical) if for all equivalences $T:{ }_{R} \mathscr{M} \rightarrow{ }_{S} \mathscr{M},{ }_{R} M \in \mathscr{A}$ implies $T(M) \in \mathscr{A}$. We remark that if $\mathscr{A}$ is a categorical class in ${ }_{R} M$, then $\Phi(\mathscr{A}), \Psi \Phi(\mathscr{A}), \Phi^{*}(\mathscr{A})$, $\Psi^{*} \Phi^{*}(\mathscr{A})$ etc. are all categorical classes. This follows from the fact that for any 
equivalence of categories $T:{ }_{R} \mathscr{M} \rightarrow{ }_{S} \mathscr{M},{ }_{R} M \succ_{R} N \Leftrightarrow{ }_{S} T(M) \succ_{S} T(N)$ and ${ }_{R} M>{ }_{R} N \Leftrightarrow{ }_{S} T(M)>{ }_{S} T(N)$.

4.3. The generating structure supported by ${ }_{R} R$. Since ${ }_{R} R$ is a generator for ${ }_{R} \mathscr{M}$, $\Psi(R)={ }_{R} \mathscr{M}$, and ${ }_{R} U \in \Phi(R)$ if and only if $U$ is a generator for ${ }_{R} \mathscr{M}$. As is well known, $U$ is a generator if and only if ${ }_{R} R$ is isomorphic to a direct summand of $U^{n}$ for some positive integer $n$.

4.4. The cogenerating structure supported by ${ }_{R} R$. For a module ${ }_{R} M$ let $M^{*}$ denote the right $R$-module $\operatorname{Hom}_{R}(M, R)_{R}$. A module ${ }_{R} M$ is called torsionless if $\psi_{M}: M \rightarrow M^{* *}$ is a monomorphism, and thus $\Psi^{*}(R)$ is the class of torsionless left $R$-modules. On the other hand, ${ }_{R} R>{ }_{R} M$ if and only if Ann $(M)=0$, that is, if and only if ${ }_{R} M$ is faithful, so $\Phi^{*}(R)$ is the class of faithful left $R$-modules.

A module ${ }_{R} P$ is projective if $P$ is a direct summand of a free module, so if $P$ is projective, then ${ }_{R} P>{ }_{R} R$. This implies that every projective module is torsionless, and also that if $\mathscr{P}$ denotes the class of projective left $R$-modules, then $\Phi^{*}(\mathscr{P})$ $=\Phi^{*}(R)$ and $\Psi^{*} \Phi^{*}(\mathscr{P})=\Psi^{*}(R)$. The classes of torsionless modules and faithful modules are therefore categorical classes of modules. The full subcategory of torsionless left $R$-modules is the inf-completion of the full subcategory of projective left $R$-modules.

4.5. The generating structure supported by ${ }_{R} E(R)$. If ${ }_{R} M$ is injective, then for each $m \in M$ there exists $f_{m}: R \rightarrow M$ defined by $f_{m}(r)=r m$, and since $M$ is injective this can be extended to $E(R)$. This shows that $E(R)>M$, so if $\mathscr{E}$ denotes the class of injective left $R$-modules, then $\Phi(E(R))=\Phi(\mathscr{E})$ and $\Psi(E(R))=\Psi \Phi(\mathscr{E})$, and these classes of modules are categorically defined. The full subcategory determined by $\Psi(E(R))$ is the sup-completion of the full subcategory of injective left $R$-modules.

4.5.1. Definition. A module ${ }_{R} M$ is called fully divisible if $E(R) \succ M$.

A module ${ }_{R} M$ is called divisible if $d M=M$ for every non-zero-divisor $d \in R$. Any direct sum of divisible modules is divisible, and a quotient of a divisible module is divisible. It can be shown that every injective module is divisible, so it follows that every module in $\Psi(E(R))$ is divisible. This motivates the above definition.

4.5.2. Proposition. Let ${ }_{R} M \in{ }_{R} \mathscr{M}$. Then $M$ is fully divisible $\Leftrightarrow$ every diagram

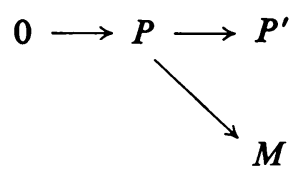

with $P$ finitely generated and projective can be completed to a commutative diagram

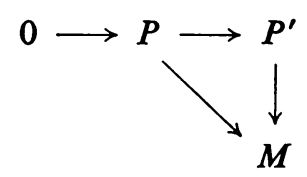


Proof. $(\Rightarrow)$. Let $i:{ }_{R} P \rightarrow{ }_{R} P^{\prime}$ be an inclusion mapping with $P$ finitely generated and projective. Suppose that $f: P \rightarrow M$ is an $R$-homomorphism and that $p: E(R)^{I} \rightarrow M$ is an epimorphism for some index set $I$. Because $P$ is projective, $f$ can be lifted to $f^{\prime}: P \rightarrow E(R)^{I}$ with $f=p f^{\prime}$, and since $P$ is finitely generated, the image of $f^{\prime}$ is contained in a finite number of components of $E(R)^{I}$, which is injective. Therefore $f^{\prime}$ can be extended to $f^{\prime \prime}: P^{\prime} \rightarrow E(R)^{I}$ with $f^{\prime \prime} i=f^{\prime}$. Then $p f^{\prime \prime} i$ $=p f^{\prime}=f$ and $p f^{\prime \prime}$ is the required extension of $f$.

$(\Leftarrow)$. By assumption every homomorphism $f: R \rightarrow M$ can be extended to $f^{\prime}: E(R) \rightarrow M$, and this is sufficient to show that $E(R)>M$.

This proposition can be used to show that a product of modules is fully divisible if and only if each component is fully divisible. It is also clear from the proposition that a finitely generated, projective and fully divisible module is injective.

The class of modules $\Phi(E(R))=\Phi(\mathscr{E})$ is dual to the class of modules $\Phi^{*}(\mathscr{P})$ $=\Phi^{*}(R)$, so the following definition seems appropriate.

4.5.3. Definition. A module ${ }_{R} M$ is called cofaithful if $M \succ E(R)$.

4.5.4. Proposition. Let ${ }_{R} M \in_{R} \mathscr{M}$. The following are equivalent:

(i). ${ }_{R} M$ is cofaithful.

(ii) There exists a finite set $\left\{m_{1}, \ldots, m_{n}\right\}$ of elements of $M$ such that Ann $\left(\left\{m_{1}, \ldots, m_{n}\right\}\right)=0$.

(iii) There exists a positive integer $n$ such that $R$ can be embedded as an $R$-submodule of $M^{n}$.

Proof. (i) $\Rightarrow$ (ii). If $M \succ E(R)$, then for $1 \in E(R)$ we must have $1=\sum_{i=1}^{n} f_{i}\left(m_{i}\right)$ for $f_{i} \in \operatorname{Hom}_{R}(M, E(R))$ and $m_{i} \in M$ for each $i$. Then if $r \in \operatorname{Ann}\left(\left\{m_{1}, \ldots, m_{n}\right\}\right)$ we must have $r \cdot 1=0$ and $r=0$.

(ii) $\Rightarrow$ (iii). If Ann $\left(\left\{m_{1}, \ldots, m_{n}\right\}\right)=0$, then the $R$-homomorphism $f: R \rightarrow M^{n}$ defined by $f(r)=\left(r m_{1}, r m_{2}, \ldots, r m_{n}\right)$ is one-to-one.

(iii) $\Rightarrow$ (i). If $i: R \rightarrow M^{n}$ is a monomorphism, then any homomorphism $f: R \rightarrow E(R)$ can be extended to a homomorphism $g: M^{n} \rightarrow E(R)$ with $g i=f$. Since $R \succ E(R)$, we must have $M^{n} \succ E(R)$ and therefore $M \succ E(R)$.

4.5.5. Corollary. A cofaithful module contains a finitely generated faithful submodule. If $R$ is commutative, then a module is cofaithful if and only if it contains a finitely generated faithful submodule.

For more detailed proofs we refer to the author's thesis [1].

We next apply Proposition 3.5 to the generating structures supported by $E(R)$ and $R$ to obtain the following result.

4.5.6. Proposition. For any ring $R$ the following are equivalent:

(i) Every cofaithful left $R$-module is a generator.

(ii) ${ }_{R} E(R)$ is a generator.

(iii) Every left $R$-module is fully divisible.

(iv) ${ }_{R} R$ is fully divisible ( $\Leftrightarrow R$ is left self-injective). 
4.6. The cogenerating structure supported by ${ }_{R} E(R)$. For each ${ }_{R} M \in_{R} \mathscr{M}$ let $T(M)$ be the intersection of the kernels of all $R$-homomorphisms from $M$ into $E(R)$. This defines the torsion radical studied by Jans [2, §3], and $M \in \Psi^{*}(E(R))$ if and only if $T(M)=0$. We fix this notation for the remainder of the paper.

By Proposition 3.11 we see that $E(R)>M$ if and only if $E(R)>\operatorname{tr}_{M}(E(R))$. We note that $\operatorname{tr}_{M}(E(R))$ is fully divisible, and that if $E(R)>\operatorname{tr}_{M}(E(R))$, then since $R>E(R)$ we must have $R>\operatorname{tr}_{M}(E(R))$, so $\operatorname{tr}_{M}(E(R))$ is faithful.

On the other hand, if ${ }_{R} R>{ }_{R} N$ and $N$ is fully divisible, then $R$ can be embedded in a product $N^{(I)}$ of copies of $N$, and since $N$ is fully divisible, so is $N^{(I)}$. This implies that the embedding can be extended to $E(R)$, and the extension is a monomorphism because $E(R)$ is essential over $R$. Thus $E(R)>N$. We have proved the following proposition.

4.6.1. Proposition. Let ${ }_{R} M \in_{R} \mathscr{M}$. The following are equivalent:

(i) $E(R)>M$.

(ii) $\operatorname{tr}_{M}(E(R))$ is faithful.

(iii) $M$ contains a faithful, fully divisible submodule.

We may now apply Proposition 3.10 to give conditions under which the cogenerating structure supported by ${ }_{R} E(R)$ coincides with that supported by ${ }_{R} R$. Proposition 4.2 can be used to show the equivalence of (iv) and (v) below, which has been obtained by Kato in [3, Proposition 1].

4.6.2. Proposition. The following are equivalent for any ring $R$ :

(i) Every faithful left $R$-module contains a faithful, fully divisible submodule.

(ii) $\operatorname{Ann}\left(\operatorname{tr}_{R}(E(R))\right)=0$.

(iii) For $_{R} M \in{ }_{R} \mathscr{M}, T(M)=0 \Leftrightarrow M$ is torsionless.

(iv) ${ }_{R} E(R)$ is torsionless.

(v) Every essential extension of a torsionless left R-module is torsionless.

4.7. The generating structure supported by ${ }_{R} K$. We will let ${ }_{R} K$ denote the direct sum of representatives from each isomorphism class of simple left $R$-module. A module ${ }_{R} M$ is called completely reducible if it is a sum of simple submodules, so in particular ${ }_{R} K$ is completely reducible. It is clear that for any module ${ }_{R} M, \operatorname{tr}_{M}(K)$ is the sum of all simple submodules of $M$, so that $\Psi(K)$ is the class of completely reducible left $R$-modules.

A module ${ }_{R} M$ is called upper-distinguished if $\operatorname{Hom}_{R}(M, R / A) \neq 0$ for each maximal left ideal $A \subseteq R$, and it follows that $M \in \Phi(K) \Leftrightarrow M$ is upper distinguished.

Proposition 3.5 can be used to give results relating this generating structure to others already studied. In particular, applying it to the generating structure supported by ${ }_{R} R$ shows that the following are equivalent: (i) every upperdistinguished left $R$-module is a generator, (ii) ${ }_{R} R$ is completely reducible, (iii) every left $R$-module is completely reducible, (iv) ${ }_{R} K$ is a generator. 
4.8. The cogenerating structure supported by ${ }_{R} K$. For ${ }_{R} M \in{ }_{R} \mathscr{M}$ let $\operatorname{Rad}(M)$ denote the intersection of all maximal submodules of $M$. It is clear that ${ }_{R} M \in \Psi^{*}(K) \Leftrightarrow \operatorname{Rad}(M)=0$, since this is the radical associated with the functor $\operatorname{Hom}_{R}(-, K)$. The notion of lower-distinguished is dual to that of upperdistinguished, and $\Phi^{*}(K)$ is the class of lower-distinguished left $R$-modules. We call $R$ semisimple if $\operatorname{Rad}(R)=0$, and applying Proposition 3.10 to the cogenerating structures supported by ${ }_{R} K$ and ${ }_{R} R$ gives the following characterization of semisimple rings.

4.8.1. Proposition. The following are equivalent:

(i) Every lower-distinguished left $R$-module is faithful.

(ii) ${ }_{R} K$ is faithful.

(iii) $\operatorname{Rad}(M)=0$ for each torsionless module ${ }_{R} M$.

(iv) $R$ is semisimple.

4.9. The cogenerating structure supported by ${ }_{R} E(K)$. It is well known that ${ }_{R} E(K)$ is the canonical injective cogenerator for the category ${ }_{R} \mathscr{M}$, so $\Psi^{*}(E(K))={ }_{R} \mathscr{M}$ and $\Phi^{*}(E(K))$ is the class of cogenerators for ${ }_{R} \mathscr{M}$. We apply Proposition 3.10 to the cogenerating structures determined by ${ }_{R} E(K)$ and ${ }_{R} E(R)$ to obtain the next result. (Recall the definition of the torsion radical $T$ in 4.6.)

4.9.1. Proposition. The following are equivalent:

(i) Every faithful, fully divisible left $R$-module is a cogenerator.

(ii) ${ }_{R} E(R)$ is a cogenerator.

(iii) $T(M)=0$ for all ${ }_{R} M \in{ }_{R} M$.

(iv) $T(E(K))=0$.

An injective left $R$-module is a cogenerator if and only if it is lower-distinguished, so (ii) above is equivalent to the statement that ${ }_{R} E(R)$ is lower-distingusihed, and since ${ }_{R} E(R)$ is essential over ${ }_{R} R$ this occurs if and only if ${ }_{R} R$ is lower-distinguished. It is well known that ${ }_{R} R$ is lower-distinguished if and only if $\imath_{R}(A)=0$ for every proper left ideal $A \subseteq R$ (see [3,2.1]) and in this case $R$ is called a right $S$-ring. Left $S$-ring is defined dually.

The ring $R$ is a left cogenerator ring if ${ }_{R} R$ is a cogenerator for ${ }_{R} \mathscr{M}$. This occurs if and only if the cogenerating structures supported by ${ }_{R} R$ and ${ }_{R} E(K)$ coincide. Furthermore, this happens if and only if the cogenerating structure supported by ${ }_{R} R$ coincides with that supported by ${ }_{R} E(R)$, and the structure supported by ${ }_{R} E(R)$ coincides with that supported by ${ }_{R} E(K)$. Choosing several statements from the many available from Proposition 4.6.2 and Proposition 4.9.1, we give the following result.

4.9.2. Proposition. The following are equivalent:

(i) ${ }_{R} R$ is a cogenerator.

(ii) Ann $\left(\operatorname{tr}_{R}(E(R))=0\right.$ and $R$ is a right S-ring. 
(iii) Every left R-module is torsionless.

(iv) Every essential extension of a torsionless left $R$-module is torsionless and every faithful, fully divisible left $R$-module is a cogenerator.

We note that if ${ }_{R} R$ satisfies the conditions of Proposition 4.6.2 and is also a left $S$-ring, then $\operatorname{tr}_{R}(E(R))=R$ and ${ }_{R} R$ must be injective. This can be employed to give further results either proved or stated by Kato [3].

In summary, we feel that the notions of generating and cogenerating structure can be used to give simple, conceptual proofs of a variety of results dealing with the homological classification of rings.

\section{BIBLIOGRAPHY}

1. J. A. Beachy, Some homological classes of rings and modules, Ph.D. Thesis, Indiana University, Bloomington, Ind., 1967.

2. J. P. Jans, Some aspects of torsion, Pacific J. Math. 15 (1965), 1249-1259. MR 33 \#163.

3. T. Kato, Torsionless modules, Tôhoku Math. J. (2) 20 (1968), 234-243. MR 38 \#182.

4. J. Lambek, Completions of categories, Lecture Notes in Math., no. 24, Springer-Verlag, Berlin and New York, 1966. MR 35 \#228.

5. J. M. Maranda, Injective structures, Trans. Amer. Math. Soc. 110 (1964), 98-135. MR 29 \#1236.

6. B. Mitchell, Theory of categories, Pure and Appl. Math., vol. 17, Academic Press, New York, 1965. MR 34 \#2647.

NORTHERN ILLINOIS UNIVERSITY,

DeKalb, Illinois 60115 